\title{
O DESAFIO DA LIDERANÇA PARA O ENFERMEIRO ${ }^{1}$
}

\author{
Ana Lúcia de Assis Simões ${ }^{2}$ \\ Neide Fávero ${ }^{3}$
}

Simões ALA, Fávero N. O Desafio da liderança para o enfermeiro. Rev Latino-am Enfermagem 2003 setembro-outubro; 11(5):567-73.

Este estudo teve como objetivos conhecer a opinião do enfermeiro sobre a possibilidade de tornar-se líder, identificar características e habilidades de um líder eficaz e apresentar pontos fortes e fracos relacionados ao desempenho da liderança, segundo a auto-avaliação dos enfermeiros. Os dados foram coletados por meio de entrevistas semi-estruturadas, realizadas com 36 enfermeiros da área hospitalar, sendo analisados segundo a técnica de análise de conteúdo. Os resultados evidenciaram que a maioria dos enfermeiros entrevistados concorda que seja possível tornar-se líder; credibilidade, comunicabilidade e conhecimentos foram características destacadas em um líder; ter bom relacionamento, envolvimento e segurança foram atitudes responsáveis pelo sucesso do enfermeiro nas experiências de liderança, caracterizando os pontos fortes, enquanto que insegurança e comunicação ineficaz representaram os pontos fracos. Concluiu-se que existem perspectivas para 0 enfermeiro melhorar seu desempenho na liderança, desde que haja mudanças no comportamento, aquisição de conhecimentos e desenvolvimento de habilidades específicas.

DESCRITORES: liderança; recursos humanos de enfermagem; equipe de enfermagem

\section{THE LEADERSHIP CHALLENGE FOR NURSES}

This study aimed at learning the opinion of nurses about the possibility of becoming a leader, identifying the characteristics and abilities of an effective leader and presenting the favorable and unfavorable points related to leadership performance, according to nurses' self-evaluation. Data were collected through semi-structured interviews with 36 hospital nurses and analyzed through the content analysis technique. Results showed that the majority of nurses agree that it is possible to become a leader; credibility, communication and knowledge were characteristics highlighted in a leader; good knowledge, involvement and security were attitudes responsible for nurses' success in their leadership experience, characterizing the favorable points and insecurity and inefficacious communication represented the unfavorable points. Authors concluded that there are perspectives for nurses to improve their performance regarding leadership but they must change their behavior, search for new knowledge and for the development of specific abilities.

DESCRIPTORS: leadership; human resources in nursing; nursing team

\section{EL DESAFÍO DEL LIDERAZGO PARA EL ENFERMERO}

Este estudio tuvo como objetivos conocer la opinión del enfermero a cerca de la posibilidad de hacerse líder; identificar características y habilidades de un líder eficaz y presentar puntos fuertes y débiles relacionados con el desempeño del liderazgo, según la auto-evaluación de los enfermeros. La recolección de datos fue hecha a través de encuestas semiestructuradas, realizadas con 36 enfermeros del área hospitalaria, analizadas mediante la técnica de análisis de contenido. Los resultados muestran que la mayoría de los enfermeros entrevistados están de acuerdo con que sea posible hacerse líder; credibilidad, comunicabilidad y conocimientos fueron características resaltadas en un líder; tener buenas relaciones, compromiso y seguridad fueron actitudes señaladas como responsables del éxito del enfermero en las experiencias de liderazgo, caracterizándolas como puntos fuertes, mientras que inseguridad y comunicación ineficaz representaron los puntos débiles. Se concluye que hay perspectivas para el enfermero mejorar su desempeño en el liderazgo, siempre y cuando haya cambios en el comportamiento, adquisición de conocimiento y desarrollo de capacidades específicas.

DESCRIPTORES: liderazgo; personal de enfermería; grupo de enfermería

\footnotetext{
${ }_{1}^{1}$ Artigo extraído da tese de doutorado "Desenvolver o potencial de liderança: um desafio para o enfermeiro", apresentada ao programa de PósGraduação em Enfermagem Fundamental da Escola de Enfermagem de Ribeirão Preto, da Universidade de São Paulo; ${ }^{2}$ Doutoranda do Programa de Enfermagem Fundamental, Docente do Centro de Graduação em Enfermagem da FMTM, e-mail: assisimoes@bol.com.br; ${ }^{3}$ Professor Titular da Escola de Enfermagem de Ribeirão Preto, da Universidade de São Paulo, Centro Colaborador da OMS para o desenvolvimento da pesquisa em enfermagem
} 
INTRODUÇÃO

Ao longo dos anos, diversas pesquisas têm enfocado o tema Liderança, buscando conceituar esse fenômeno; relacionar características e habilidades presentes nas pessoas que se destacam como líderes; diferenciar estilos comportamentais, valorizar aspectos situacionais, enfim, ressaltar as diferentes variáveis que podem intervir nesse processo.

Em 1976, a partir de uma síntese de vários conceitos, a liderança é definida como o processo de exercer influência sobre um indivíduo ou um grupo, em uma dada situação, nos esforços para a consecução de objetivos comuns ${ }^{(1)}$.

Numa perspectiva contemporânea ${ }^{(2)}$, há uma concordância em que a liderança seja um fenômeno grupal e que envolve um sistema de influência social de um indivíduo sobre os demais; e, ainda, ser um processo coletivo, compartilhado entre os elementos de um grupo.

Diferentemente dos estudos iniciais, onde a ênfase era dada à pessoa e ao poder detido pelo líder, atualmente observamos que a marca da liderança moderna é fortalecer o grupo de trabalho, ressaltando e valorizando as competências individuais, diluindo o poder na equipe, fazendo com que cada membro reconheça o propósito e o significado de seu trabalho. Por meio da denominação "liderança transformadora", à ação, converte seguidores em líderes, e líderes, em agentes de mudança.

Nessa perspectiva, o enfermeiro deve estar orientado para as possibilidades de desempenhar esse novo papel de líder, mais orientado para o futuro, mais flexível, dinâmico e disposto a assumir riscos, em contraposição ao papel controlador, ditador de regras, normas e procedimentos.

A esse respeito, enfatiza-se que os enfermeiros devem estar dispostos a rejeitar a rotina, confrontar questões e implementar ações que levem a mudanças ${ }^{(4)}$.

O desafio de mudar o perfil do administrador do cuidado à saúde requer o desenvolvimento e a maturação de novos tipos de liderança, os quais devem estar menos voltados para o dar ordens, papel que traduz o paradigma do padrão administrativo vigente na era da Revolução Industrial. Ressalta-se, ainda, que os novos líderes não dão ordens, apenas motivam as pessoas a agirem ${ }^{(5)}$.

A era do chefe controlador é substituída pela do líder coordenador, cabendo a este interpretar o sentido daquilo que existe de imaginário nas expectativas dos seguidores, juntamente com o que existe de simbólico na cultura organizacional ${ }^{(6)}$.

Nessa nova era, o papel dos seguidores é tão importante quanto o do próprio líder. Não é mais concebível falar em líder sem se lembrar dos seguidores, pois, "sem seguidores, simplesmente não há liderança"(7). Por essa razão, o vínculo entre líder e seguidor é considerado uma relação simbiótica ${ }^{(8)}$.

Líderes não são um sucesso por si mesmos, pois utilizam outras pessoas enquanto estabelecem e administram o trabalho na organização ${ }^{(9)}$; há que se lembrar de que, na enfermagem, não seria possível proporcionar uma assistência de qualidade sem o suporte, a cooperação e a contribuição dos seguidores ${ }^{(8)}$.

Lembramos que a posição formal ocupada pelo enfermeiro no organograma institucional não lhe garante o poder de líder, pois o que autoriza alguém a agir como líder é a percepção positiva que os seguidores apresentam em relação à pessoa ${ }^{(6)}$. Nessa direção, recomenda-se que a liderança seja vista como uma responsabilidade e não como posição e privilégios ${ }^{(10)}$.

É oportuno, também, mencionar que a liderança transcende cargos ou posições formais, não carecendo de institucionalização, pois é decorrente de uma sintonia espontânea e informal estabelecida entre líderes e seguidores $^{(11)}$

Pesquisas já realizadas ${ }^{(12)}$ demonstram características de líderes freqüentemente admiradas pelos seguidores, sendo que honestidade, competência, olhar para diante e inspiração encabeçam a lista de atributos. De acordo com os autores, esses atributos podem ser resumidos em um único termo: credibilidade.

Credibilidade representa um dos três contextos principais da liderança (ao lado de comprometimento e complexidade), sendo que, nos dias de hoje, o fato de ter crédito é considerado pelos autores como uma premiação, dadas as exigências e desafios por que passam os líderes ${ }^{(3)}$.

O que mais as pessoas esperam de um líder é que ele desperte confiança, que seja digno de crédito, demonstrando coerência com a filosofia empresarial e congruência em suas idéias, discurso e ações; a lealdade e o comprometimento dos seguidores podem depender da credibilidade percebida ${ }^{(12)}$.

Compartilhando dessa opinião, ressalta-se que ganhar a confiança dos seguidores é um requisito essencial 
para a liderança eficaz, e, para isso, ações e crenças professadas devem ser congruentes ${ }^{(10)}$.

Em tempos de mudanças e de oportunidades, os grupos de trabalho devem estar alinhados, firmes em propósitos comuns e direções, para alcançar objetivos estratégicos, sendo que, para isso, a liderança é necessária ${ }^{(5)}$.

Frente ao exposto, entendemos que, na enfermagem, torna-se cada vez mais urgente a necessidade de enfrentar as mudanças, de serem criadas novas perspectivas de trabalho, com aquisição de novos conhecimentos e habilidades, a fim de que o enfermeiro apresente um melhor desempenho de suas funções, sejam elas assistenciais ou administrativas.

Um dos desafios atuais encontrados pela enfermagem é conseguir oferecer um atendimento de melhor qualidade ao cliente, conciliando uma diminuição dos custos desse serviço. Para enfrentar esse desafio, recomenda-se que novos caminhos sejam pesquisados e que os líderes redefinam a essência da arte e ciência da enfermagem, buscando desenvolver estratégias que levem a uma prática profissional de maneira mais holística ${ }^{(13)}$.

Complementando essa idéia, a principal função do enfermeiro líder é criar e apoiar uma prática voltada para um cuidado mais humanitário, sensitivo e atencioso, enfocando as necessidades individuais do cliente ${ }^{(14)}$.

Quanto ao desempenho da liderança, são apontadas dificuldades freqüentemente encontradas pelos enfermeiros, as quais se referem às questões de relacionamento na equipe e às próprias atribuições desses profissionais $^{(15)}$. Além das diversas dificuldades em liderar a equipe de enfermagem, merece destaque a insatisfação apresentada em relação à formação profissional, especificamente quanto ao preparo para o exercício da liderança; ressalta-se a importância de se oferecer oportunidade para o aluno desenvolver atitudes críticas e reflexivas, a criatividade e a consciência de que devem atuar como agentes de mudança ${ }^{(15)}$.

Considerando a importância, para o enfermeiro, de conseguir um melhor desempenho em relação à liderança da equipe de enfermagem, realizamos esse estudo com os seguintes objetivos: 1) conhecer a opinião do enfermeiro sobre a possibilidade de tornar-se um líder; 2) identificar, segundo a opinião dos enfermeiros, as principais características e habilidades de um líder; 3) apresentar, de acordo com a auto-avaliação dos enfermeiros, pontos fortes e fracos relacionados ao desempenho da liderança.

\section{PROCEDIMENTOS METODOLÓGICOS}

O estudo foi realizado em um hospital público federal, localizado na microrregião do Triângulo Mineiro, no estado de Minas Gerais. Trata-se de um hospital geral, de grande porte, destinado a desenvolver e apoiar as atividades de ensino, pesquisa e extensão, além da prestação de serviços assistenciais à população.

Fizeram parte da amostra, 36 enfermeiros, lotados nos diversos setores do hospital, os quais são responsáveis pela organização do serviço e coordenação das equipes de enfermagem.

Os dados foram coletados por meio da aplicação de um questionário, para o qual foi elaborado um roteiro, com as seguintes questões norteadoras: Na sua opinião, é possível tornar-se líder? (Justifique sua resposta); Quais características e habilidades você reconhece em uma pessoa considerada um líder eficaz?; Destaque, das suas experiências de liderança, pontos fortes e fracos relacionados ao seu desempenho.

Posteriormente, o material apreendido foi submetido aos procedimentos de análise ${ }^{(16)}$. As respostas dadas a uma mesma pergunta foram classificadas por temas, resultando na listagem das principais informações contidas nos questionários.

Ressaltamos que o projeto foi aprovado pelo Comitê de Ética em Pesquisa e que os participantes foram esclarecidos sobre os objetivos e a finalidade da pesquisa, dando o consentimento para a sua participação.

\section{APRESENTAÇÃO E DISCUSSÃO DOS RESULTADOS}

Passamos a apresentar os resultados da análise, procurando descrever os aspectos da situação estudada, julgados como os mais significativos para os enfermeiros.

Quando indagados sobre a possibilidade de tornarse líder, 28 enfermeiros responderam afirmativamente, justificando suas respostas, conforme evidenciam as falas que expressam suas opiniões sobre essa possibilidade:

...com mudança de ações e pensamentos.../...adquirindo conhecimentos..

...desenvolvendo habilidades.../...descobrindo potencialidades...

...por meio de experiências.......buscando o aperfeiçoamento.../...basta querer... 
...com coragem e força de vontade...

...com iniciativa, confiança, competência, envolvimento, dedicação...

As falas acima permitem afirmar que a grande maioria dos participantes concorda com a possibilidade de vir a ser um líder a partir do desenvolvimento do potencial latente que existe nas pessoas. Tais opiniões têm justificativas pertinentes, conforme dados da literatura, que recomendam promover o autoconhecimento, vivenciar experiências de liderança e buscar um aprendizado significativo, como estratégias imprescindíveis para quem deseja desenvolver-se na liderança ${ }^{(12,17-18)}$.

Por outro lado, ainda que em menor número, alguns enfermeiros afirmaram não acreditar que seja possível tornar-se um líder, alegando que a liderança depende de características pessoais natas, de possuir o dom, ou seja, concordam em que um líder já nasce líder. Tal entendimento encontra-se vinculado às primeiras compreensões desse fenômeno, quando se acreditava que a liderança era inata, momento em que foi estabelecido um conjunto de traços pessoais que definiam o perfil do líder ideal, distinguindo-o das demais pessoas ${ }^{(6)}$.

As falas a seguir revelam esse posicionamento:

...o líder é nato......tem que ter espírito de liderança.../ ...características pessoais inatas...

...é um dom.../...depende da aceitação do grupo...

A esse respeito, é importante comentar que nenhuma mudança ocorre, seja na dimensão individual ou coletiva, sem que haja uma predisposição, um motivo que incite as pessoas à ação; principalmente no âmbito da liderança, por envolver uma série de comportamentos específicos, de habilidades próprias e de experiências de vida.

A literatura sinaliza um crescente consenso a respeito de atributos identificáveis em líderes eficazes, denominados habilidades aprendidas, atitudes desenvolvidas e valores, os quais se sobressaem às características pessoais estáveis.

O desenvolvimento das habilidades de um líder ocorre à medida que o indivíduo permite a penetração e o amadurecimento de novas idéias em sua mente, dedicando-se ao aprendizado da liderança e esforçandose para enriquecer a própria experiência ${ }^{(2)}$.

Assim, as pessoas devem criar suas próprias agendas de aprendizado, encontrando as oportunidades para desenvolver suas forças e vencer suas fraquezas ${ }^{(12)}$. Vale acrescentar que a crença na impossibilidade de aprender a liderar é um impedimento para o desenvolvimento da liderança, muito mais poderoso que a própria natureza desse fenômeno ${ }^{(12)}$.

Destaca-se, ainda, que investir no desenvolvimento e na recompensa de enfermeiros líderes constitui uma boa estratégia para aumentar a qualidade do cuidado ao cliente e para melhorar o relacionamento interdisciplinar, a imagem e o status da enfermagem ${ }^{(14)}$.

Estimulados a pensar em pessoas reconhecidas como líderes eficazes e nas características e habilidades apresentadas por elas, os enfermeiros foram enfáticos em relacionar diversos atributos considerados necessários para um líder. Uma leitura atentiva desses atributos possibilitou a classificação conforme mostra a Tabela 1.

Tabela 1 - Freqüência das características e habilidades do líder eficaz, segundo a opinião dos enfermeiros pesquisados - Uberaba, 2001

\begin{tabular}{l|c}
\hline \multicolumn{1}{c|}{ CARACTERISTICAS E HABILIDADES } & FREQÜÊNCIA \\
\hline Comunicabilidade & 25 \\
Conhecimento/Experiência & 24 \\
Honestidade & 19 \\
Integridade & 15 \\
Determinação & 13 \\
Responsabilidade & 12 \\
Influência & 11 \\
Participação & 10 \\
Segurança & 09 \\
Coragem & 06 \\
Criatividade & 06 \\
Iniciativa & 03 \\
\hline
\end{tabular}

A análise desses dados evidencia que possuir habilidade de comunicação foi o aspecto mais lembrado em um líder, obtendo a maior freqüência de indicação pelos participantes da pesquisa, seguida pela posse de conhecimentos e experiências.

Em seguida, destacaram-se características como honestidade, integridade, determinação e responsabilidade, as quais foram citadas repetidas vezes nas respostas. Vale mencionar que o conjunto desses atributos $^{(3,12)}$ recebe a nomeação credibilidade, o que é considerado, pelos autores, o aspecto mais admirado em um líder.

Essa constatação reforça a opinião sobre o que mais se espera de um líder, ou seja, que seja digno de crédito, podendo confiar na existência de coerência entre suas palavras e ações ${ }^{(12)}$. Acrescenta-se que o líder só conquista a aquiescência do grupo para dirigi-lo, passando a ser digno de crédito, a partir do momento em que é percebido de maneira favorável pelos seguidores ${ }^{(6)}$.

Ainda em relação às características e habilidades reconhecidas no líder eficaz, destacaram-se o poder de 
exercer influência nas pessoas e o estilo participativo de liderança. É oportuno lembrar que a capacidade de influenciar pessoas é um dos aspectos básicos das definições de liderança.

Nesse contexto, vale dizer que, embora nenhum traço em particular garanta o sucesso de um líder, muitas características são potencialmente úteis na distinção entre líderes eficazes e outras pessoas ${ }^{(19)}$.

Com o intuito de levá-los a refletir sobre as próprias experiências de liderança, sugerimos aos enfermeiros que tentassem relembrar algumas situações que exigiram o desempenho do papel de líder, e que destacassem, dessas experiências vividas, pontos fortes e pontos fracos. Analisando os relatos dos enfermeiros, foi possível abstrair informações possíveis de serem classificadas como PONTOS FORTES E PONTOS FRACOS.

No item PONTOS FORTES, foram agrupadas opiniões que demonstram comportamentos que levaram ao sucesso do enfermeiro como líder; em PONTOS FRACOS, encontram-se listadas as posturas que foram prejudiciais ao desempenho da liderança na experiência vivida. Na Tabela 2, encontram-se especificados quais foram os comportamentos responsáveis pelo sucesso ou pelo insucesso das experiências de liderança, vividas pelos enfermeiros entrevistados.

Tabela 2 - Freqüência dos PONTOS FORTES e PONTOS FRACOS, destacadas das experiências de liderança, segundo a opinião dos enfermeiros pesquisados - Uberaba, 2001

\begin{tabular}{lclc}
\hline PONTOS FORTES & FREQÜENCIA & PONTOS FRACOS & FREQÜẼNCIA \\
\hline Bom relacionamento & 14 & Insegurança & 27 \\
Envolvimento & 12 & Comunicação ineficaz & 11 \\
Segurança & 10 & Resolução de conflito & 04 \\
Comunicação eficaz & 07 & & \\
Iniciativa & 07 & & 42 \\
\hline TOTAL & 50 & TOTAL & \\
\hline
\end{tabular}

Como demonstrado na Tabela 2, as condições que determinaram a eficácia da liderança do enfermeiro em algumas experiências vivenciadas, seus pontos fortes, estiveram atreladas a comportamentos geradores de bom relacionamento, como exemplificam as falas:

...transmitindo confiança ao grupo.......por meio do bom relacionamento com as pessoas...

...sabendo trabalhar com pessoas diferentes.../...estando aberta ao grupo...

Não há dúvidas de que existe uma relação recíproca entre relacionamento interpessoal e sucesso da liderança. Líderes competentes encorajam e promovem a auto-suficiência, o pensamento crítico, a individualidade e a aprendizagem contínua, de modo a contribuir com a formação de seguidores exemplares, tendo em vista o sucesso do líder, da organização, do próprio grupo e da profissão( ${ }^{(8)}$.

Estar envolvido com o trabalho foi a segunda condição determinante do êxito da experiência de liderança, na opinião dos enfermeiros. Para eles, o envolvimento, o amor ao que se faz, o entusiasmo e o otimismo são fatores que podem contribuir para o bom desempenho de um líder.

É interessante observar que a habilidade de comunicação foi referenciada nos dois extremos, ou seja, dependendo da maneira como foi praticada, determinou o sucesso ou o fracasso da liderança. Quando se estabelece a comunicação de forma eficaz, com transparência e expressão clara de objetivos para a equipe, suas conseqüências são positivas para a experiência de liderança, configurando como "ponto forte". Por outro lado, se existe dificuldade em expressar-se ou falta clareza nas colocações, a experiência de liderança estará fadada ao insucesso, caracterizando um ponto fraco.

Certamente, uma boa comunicação, onde há transmissão de mensagens de forma clara e objetiva, proporciona um ambiente de trabalho produtivo e harmonioso, num clima de confiança e credibilidade ${ }^{(20)}$.

Porém, o grande destaque como ponto fraco nas experiências de liderança, conforme apresentado na Tabela 2, foi a postura de insegurança, a qual mostrou ser responsável pela maioria dos fracassos dos enfermeiros como líderes da equipe de enfermagem. As falas selecionadas abaixo retratam essa situação:

...medo de assumir riscos.../...a falta de experiência..../ ...medo da responsabilidade...

...a timidez...a ansiedade.../...a falta de conhecimentos...

Ficou caracterizado que a falta de conhecimentos e de experiências no trabalho, o medo de assumir riscos, o medo das incertezas e a dificuldade em enfrentar desafios, geram o sentimento de insegurança no enfermeiro, em determinadas situações, influenciando, sobremaneira, na possibilidade de liderar a equipe de enfermagem. Evidentemente, tais posturas não fazem parte do rol de habilidades que caracterizam um líder eficaz.

Em contraposição, o fato de ter segurança foi registrado na categoria PONTO FORTE, ocupando o terceiro lugar nas citações dos enfermeiros. Esse fato reforça a importância e a necessidade de possuir 
conhecimentos, ter boa argumentação, apresentar firmeza nas decisões e manter claras convicções, para o sucesso da liderança.

Destacaram-se, ainda, como ponto forte, a capacidade de iniciativa, representada pelas falas implementação de novas idéias e vontade de fazer as coisas; como ponto fraco, a dificuldade na resolução de conflitos, manifestada pelas seguintes verbalizações: dificuldade em lidar com conflitos entre as pessoas e estar à frente quando há resistência dos membros da equipe.

Essas colocações remetem ao fato de que o desempenho da liderança está associado a competências específicas, classificadas em dimensões distintas, referentes ao domínio do contexto organizacional, às habilidades interpessoais e às características individuais, as quais são responsáveis pelo sucesso de um líder ${ }^{(2)}$.

Frente ao exposto, reconhecemos que, para o enfermeiro, desempenhar a função de líder da equipe da enfermagem é uma experiência que, muitas vezes, encontra-se permeada por dificuldades decorrentes da inabilidade em lidar com situações grupais, da falta de conhecimentos profissionais e da incapacidade de manter uma comunicação eficaz.

\section{CONSIDERAÇÕES FINAIS}

Autores contemporâneos compartilham a opinião de que é possível a aprendizagem da liderança, pois a mesma compreende uma função gerencial que requer qualidades individuais e habilidades específicas, passíveis

\section{REFERÊNCIAS BIBLIOGRÁFICAS}

1. Hersey $\mathrm{P}$, Blanchard $\mathrm{KH}$. Psicologia para administradores de empresas. São Paulo (SP): EPU/MEC; 1976.

2. Motta PR. Gestão contemporânea: a ciência e a arte de ser dirigente. 9a ed. Rio de Janeiro (RJ): Record; 1998.

3. Bennis W, Nanus B. Líderes: estratégias para assumir a verdadeira liderança. São Paulo (SP): Harbra; 1988.

4. Valiga TM. Leadership for the future. Holist Nurs Pract 1994; 9 (1): 83-90.

5. Filson B. The new leadership. Hospitals \& Health Networks 1994 Sept; 76.

6. Bergamini CW. O desafio da liderança. In: Bergamini CW, Coda R, organizadores. Psicodinâmica da vida organizacional: motivação e liderança. $2^{a}$ ed. São Paulo (SP): Atlas; 1997. p. 319-42.

7. Kets de Vries MFM. Liderança na empresa: como o comportamento dos líderes afeta a cultura interna. São Paulo (SP): Atlas; 1997.

8. Di Rienzo SM. A challenge to nursing: promoting followers as well as leaders. Holist Nurs Pract 1994; 9(1):26-30. de serem aprendidas por todos os indivíduos ${ }^{(2-3,12)}$.

Os resultados encontrados neste estudo demonstraram que a maioria dos enfermeiros concorda com a possibilidade de tornar-se líder, sinalizando para o desenvolvimento da liderança do enfermeiro, a partir de uma maior iniciativa, envolvimento e vontade de mudar. Descobrir as próprias potencialidades, aproveitar as experiências do trabalho e buscar aperfeiçoar-se profissionalmente configuram oportunidades para esse desenvolvimento, de acordo com as justificativas dos enfermeiros entrevistados.

Dentre os atributos de um líder eficaz, a credibilidade destacou-se como um quesito fundamental, relacionada a comportamentos que denotam honestidade, integridade e dinamismo, constatando que, para se conseguir o crédito dos seguidores, é necessário existir coerência entre ações e palavras do dirigente.

As experiências profissionais desses enfermeiros revelaram que estabelecer um bom relacionamento com os funcionários, estar envolvido com as questões do trabalho, demonstrar segurança na condução da equipe e manter uma comunicação eficaz são condições importantes para o sucesso na liderança do enfermeiro.

Sendo assim, almejar melhor desempenho do enfermeiro, como líder da equipe de enfermagem, pressupõe a ocorrência de mudanças de comportamentos, de busca constante de novos conhecimentos e do desenvolvimento de habilidades essenciais para a liderança, as quais envolvem qualidades pessoais, habilidades interpessoais e o domínio do contexto organizacional $^{(2)}$.

9. Hrezo RJ, Witte RE. Leadership: moving into the twenty-first century. Nurs Manage 1993; 24(5).

10. Drucker PF. Administrando para o futuro: os anos 90 e a virada do século. $5^{\underline{a}}$ ed. São Paulo (SP): Pioneira; 1996.

11. Srour RH. Poder, cultura e ética nas organizações. Rio de Janeiro (RJ): Campus; 1998.

12. Kouzes JM, Posner BZ. O desafio da liderança: como conseguir feitos extraordinários em organizações. Rio de Janeiro (RJ): Campus; 1991.

13. Vautier AF. Guest Editorial. Nurs Adm 1997; 21(2):vii-ix. 14. Binnie A. How to grow more leaders. Nurs Times 1998 July; 94(28):24-6.

15. Simões ALA. O ser líder no cotidiano do profissional enfermeiro. [Dissertação]. Ribeirão Preto (SP): Escola de Enfermagem de Ribeirão Preto/USP; 1997.

16. Thiollent M. Pesquisa-ação nas organizações. São Paulo (SP): Atlas; 1997.

17. Dunhan-Taylor J, Fisher E, Kinion E. Experiences, events, peoples: do they influence the leadership style of nurse executives? JONA 1993 July/Aug; 23(7/8):30-4. 
18. Allen DW. How nurses become leaders: perceptions and beliefs about leadership development. JONA 1998 Sept; 28(9):15-20.

19. Bateman TS, Snell SA. Administração: construindo vantagem competitiva. São Paulo (SP): Atlas; 1998.

20. Quick TL. Como desenvolver equipes bem-sucedidas.

Rio de Janeiro (RJ): Campus; 1997. 


\title{
A FORMAČ̃O DO ENFERMEIRO CRÍTICO-REFLEXIVO NO CURSO DE
} ENFERMAGEM DA FACULDADE DE MEDICINA DE MARÍLIA - FAMEMA

\author{
Mara Quaglio Chirelli \\ Silvana Martins Mishima²
}

Chirelli MQ, Mishima SM. A formação do enfermeiro crítico-reflexivo no Curso de Enfermagem da Faculdade de Medicina de Marília - FAMEMA. Rev Latino-am Enfermagem 2003 setembro-outubro; 11(5):574-84.

Objetivou-se neste estudo, captar através dos alunos do Curso de Enfermagem da FAMEMA como está sendo construído seu processo de formação, visando a constituição de um profissional crítico-reflexivo, e identificar as marcas diferenciais deste processo percebidas pelos alunos a partir da lógica do Projeto Político-Pedagógico (PPP). Os sujeitos da pesquisa foram alunos do $4^{\circ}$ ano do Curso de Enfermagem, sendo utilizados o grupo focal e a entrevista semi-estruturada como técnicas de pesquisa. O material empírico foi organizado segundo a técnica do Discurso do Sujeito Coletivo, e procedeu-se em seguida à análise temática. Evidenciou-se que os alunos conseguiram elaborar uma crítica com relação ao PPP, discriminando e percebendo problemas apontados, mudanças que ocorreram, conflitos gerados num projeto dessa natureza. Ao vivenciarem a realidade concreta, os alunos também convivem com os conflitos que permeiam as relações construídas no cotidiano, questionando o papel do enfermeiro, refletindo sobre sua postura ética, a fundamentação para a argumentação e a sustentação de sua prática na equipe.

DESCRITORES: educação em enfermagem; currículo; enfermagem

\section{CRITICAL REFLEXIVE EDUCATION OF NURSES FROM THE NURSING UNDERGRADUATE PROGRAM OFFERED BY THE SCHOOL OF MEDICINE OF MARILIA-FAMEMA}

The study aimed at learning with the undergraduate students enrolled at the Nursing Undergraduate Program offered by FAMEMA about how they are building their education with the purpose of forming a critical reflexive professional as well as identifying the differences perceived by the students with respect to the logic of the Pedagogical Political Plan. The subjects were students finishing the fourth year of the undergraduate program. Authors used the focal group and semi-structured interviews as research techniques. The empirical data were organized according to the technique of Collective Subject Discourse followed by thematic analysis. Authors evidenced that the students were able to elaborate a critique with respect to the Pedagogical Plan, discriminating and perceiving the problems, the changes that occurred and the conflicts generated in a Project of this nature. When they experience reality, students also have conflicts that permeate the relationships they build in their lives, questioning nurses' role, reflecting about their ethical posture, the basis for the argumentation and support to their practice working as a team.

DESCRIPTORS: nursing education; curriculum; nursing

\section{LA FORMACIÓN DEL ENFERMERO CRÍTICO-REFLEXIVO EN EL CURSO DE ENFERMERÍA DE LA FACULTAD DE MEDICINA DE MARÍLIA - FAMEMA}

Se pretendió en este estudio, identificar a través de los estudiantes del Curso de Enfermería de la FAMEMA como está siendo su proceso de formación en la construcción de un profesional crítico-reflexivo e identificar las marcas diferenciales de este proceso identificada por los estudiantes a partir de la lógica del Proyecto Político Pedagógico (PPP). El grupo de investigación fueron los estudiantes de 4 año del Curso de Enfermería, usándose el grupo focal y la entrevista semi-estructurada como técnicas de la investigación. El material empírico fue organizado según la técnica del Discurso del Sujeto Colectivo y después se realizó el análisis temático. Se evidenció que los estudiantes consiguieron elaborar una crítica en relación con el PPP, percibiendo y diferenciando los problemas que notaron, los cambios que ocurrieron y los conflictos generados en un proyecto de esta naturaleza. Al vivir esta realidad concreta, los estudiantes también conviven con los conflictos que se desarrollan en las relaciones construidas en lo cotidiano, cuestionando el papel de la enfermera, reflexionando sobre su postura ética, los fundamentos para sustentar y argumentar su practica en equipo.

DESCRIPTORES: educación en enfermería; currículo; enfermería

\footnotetext{
${ }^{1}$ Enfermeiro, Professor Assistente junto ao Curso de Enfermagem da Faculdade de Medicina de Marília, Doutoranda do Programa Interunidades de Enfermagem das Escolas de Enfermagem da Universidade de São Paulo, e-mail: chirelli@terra.com.br; ${ }^{2}$ Enfermeiro, Professor Doutor da Escola de Enfermagem de Ribeirão Preto, da Universidade de São Paulo, Centro Colaborador da OMS para o desenvolvimento da pesquisa em enfermagem, e-mail: smishima@eerp.usp.br
} 